\title{
Cylindrical Dielectric Resonator Antennas with Harmonic Control as an Active Antenna Radiator
}

\author{
L. Lucci, ${ }^{1}$ G. Manara, ${ }^{2}$ P. Nepa, ${ }^{2}$ G. Pelosi, ${ }^{1}$ and S. Selleri ${ }^{1}$ \\ ${ }^{1}$ Department of Electronics and Telecommunications, University of Florence, Via C. Lombroso 6/17 - 50137 Firenze, Italy \\ ${ }^{2}$ Department of Information Engineering, University of Pisa, Via G. Caruso 16, 56126 Pisa, Italy
}

Correspondence should be addressed to L. Lucci, leonardo.lucci@unifi.it

Received 30 September 2008; Accepted 19 January 2009

Recommended by Giovanni Toso

A cylindrical dielectric resonator antenna is proposed as a radiator for an active integrated antenna. Harmonic tuning, which is the key step in designing active antenna radiators, is achieved via a combination of shape factor control over the resonator and insertion of reactive elements in the feed system. Numerical simulations are carried out in a finite elements framework and a layout for the complete antenna is proposed, aimed at compactness for subsequent utilization of the radiator as an element in an active array for satellite communications.

Copyright () 2009 L. Lucci et al. This is an open access article distributed under the Creative Commons Attribution License, which permits unrestricted use, distribution, and reproduction in any medium, provided the original work is properly cited.

\section{Introduction}

Active integrated antennas (AIAs) are microwave systems which integrate on a single substrate several functions, at least one of which is that of a radiating element and another is that of an active device, be it a power amplifier for a transmitting AIA, or a low noise amplifier for a receiving AIA.

Albeit more complex functions can be embedded in the AIA, as frequency conversions with its own local oscillator, phase locking and many more, the key feature of an AIA is the tight integration between the active device and the radiating element.

Conventional antenna system design leads to radiators whose input impedance is matched to $50 \Omega$ via some appropriate matching network, and to amplifier which are themselves matched to the same $50 \Omega$ via some appropriate matching network. The two devices are then connected via a $50 \Omega$ transmission line. The two matching network can be fairly complex, especially the one which must be designed for a power amplifier (PA). In this case the matching network needs not only to match the output of the PA to $50 \Omega$ at the working frequency, but must also provide an appropriate load for the higher harmonics. The essence of AIAs is to eliminate both matching networks and the interconnecting line, by designing the radiating element so that it provides the correct load to the PA at the fundamental frequency and higher harmonics. In transmission mode, in particular, the antenna should provide a given, generally complex, load for the power amplifier at the system working frequency in order to optimize the system performance, while for the first two higher harmonics, the impedance must be typically purely imaginary, be it capacitive or inductive.

By attaining this the overall system is simplified and reduced in size, by removing two matching networks, the losses in the lines are of course reduced, hence higher efficiencies, and lower power consumptions, attained. These characteristics make AIAs very attractive in wireless communications. The possibility of integrating also some sort of phase control in the AIA, for example, with a voltage controlled oscillator (VCO) leads to very attractive possibilities in the field of smart antennas and electronically steering phased arrays for vehicle-to-satellite broadband communications $[1,2]$.

As stated before AIAs are devices in which the radiating element and the active circuit, the amplifier, are treated as a single entity. The radiating element, which will be the focus of this paper, is to be seen as a filter, output matching circuit, and harmonic tuner in addition to its characteristics of load and radiating element. The core issue is that radiating elements are either broad band or resonating structures. 
Broadband antennas are usually large and unsuitable for commercial telecommunications usage, since standards give fairly narrow bands. Resonating antennas are much more compact but, being resonant, have the strong tendency to behave in a very similar way at the working frequency and at its harmonics, at least for what concerns the input impedance. This implies that higher harmonics have an input impedance which is essentially real. To allow for a resonant radiator to be embedded in an AIA some modifications are necessary to attain its harmonic tuning, that is, the separate design of the input impedance at the fundamental frequency and at at least the first two higher harmonics.

In literature there are several works presenting different patch and slot antenna configurations for harmonic tuning, most of them relies on the suppression of higher resonances. Rectangular patches can be loaded with shortening posts [3] or with notches [4] or by feeding them with photonic band-gap structures [5], while circular ones can have a slice chopped of as in [6]. Slot antennas can be loaded with reactive components shortening higher frequencies, as in $[7,8]$, leading to excellent harmonic suppression, but slot antennas tend to have poor radiating characteristics.

In this paper a harmonic tuning technique for a dielectric resonator antenna (DRA) working in $\mathrm{Ku}$ band at $14.25 \mathrm{GHz}$ is described. DRAs are a class of antennas which has interesting characteristics for usage as an AIA radiator. They exhibit low losses and, if fed via ground plane slot coupling, allow for a decoupling between the DRA, which lies on one side of the ground plane, and the substrate with its circuits and active elements, which lies on the other side of the ground plane. The absence of a substrate on the radiating side has additional benefits in array configurations, eliminating the possible interantenna coupling due to surface wave excitation.

In literature several kinds and shapes of DRAs have been considered, rectangular (RDRA), cylindrical (CDRA), hemispherical (HDRA) and all of these received much attention for what concerns antenna efficiency, compactness [911], and bandwidth. Nevertheless, there have been very few studies regarding the behavior of DRAs at higher harmonics for their usage as AIAs. More recent papers are focused on RDRA harmonic tuning $[12,13]$ while few preliminary results on CDRA harmonic tuning were presented in [14, 15].

Here a study of a slot-coupled cylindrical DRA configuration is presented, analyzing its harmonic response first at a shape factor level, then by introducing reactive elements in the coupling slot, and lastly by introducing additional slots. A complete design ready to be integrated with a PA is then presented.

The paper is organized as follows. In Section 2 the slotcoupled CDRA is described, with particular attention to higher-order resonant modes, to devise a possible optimal shape factor. Section 3 will present two modified configurations for the optimization of the resonator antenna electromagnetic performance at fundamental frequency and higher harmonics. Section 4 will present the final design suitable for the integration with a power amplifier and Section 5 will draw some conclusions.

\section{Shape Factor Tuning}

A first investigation is done by analyzing the modes which are theoretically present on a cylindrical resonator of radius $a$ and height $2 d$ made by a dielectric characterized by a permittivity $\varepsilon$ and a permeability $\mu$. The resonant frequencies are [16]

$$
f_{n m p}=\frac{1}{2 \pi a \sqrt{\mu \varepsilon}} \sqrt{\left[X_{n p} \mid X_{n p}^{\prime}\right]^{2}+\left(\frac{\pi a}{2 d}(2 m+1)\right)^{2}},
$$

where $n=1,2,3, \ldots, m=1,2,3, \ldots$, and $p=0,1,2, \ldots$ are three integer numbers, and $\left[X_{n p} \mid X_{n p}^{\prime}\right]$ are either the $p$ th zeros of the Bessel function of the first kind and order $n$ or the zeros of the derivative of the Bessel function of the first kind and order $n$, respectively.

If the zeros of the Bessel function are used the frequencies of the $T E_{n m p}$ modes are obtained. If the zeros of the derivative are used then the $T M_{n m p}$ modes are obtained. It is easy to obtain that the fundamental mode in a cylindrical resonator is the $T M_{110}$, which is relevant to $X_{11}^{\prime}=1.841$.

It is then quite easy to invert (1) so as to obtain the value of $a$ as a function of $d$ which leads to a desired fundamental mode frequency $f_{0}$ :

$$
a=\frac{X_{11}^{\prime} 2 d}{\pi \sqrt{4\left(f_{0} / c\right)^{2}(2 d)^{2}-1}}
$$

being $c=1 / \sqrt{\varepsilon \mu}$ the speed of light in the dielectric.

By choosing an appropriate range for $2 d$ and by computing the relevant values of $a$ via (2) a set of resonators whose fundamental mode frequency is $14.25 \mathrm{GHz}$ is obtained. By applying on this set (1) the frequencies of all the other modes can be computed as a function of $2 d$. Figure 1 shows such a diagram, presenting the values of $2 d$ on the $y$ axis and the frequencies on the $x$ axis. TE modes are represented with solid lines, while TM modes represented with dashed lines. It is apparent how the fundamental frequency, being a vertical line, does not vary with $2 d$. In this preliminary analysis a relatively low permittivity resonator $(\varepsilon=9.8)$ is used.

While the fundamental mode is of course fixed at the desired value independently of $2 d$, nearly all other modes are $2 d$ dependent. The first step will then be that of choosing $2 d$ so that no modes are present at the harmonics of the fundamental frequencies $2 f_{0}$ and $3 f_{0}$, represented with pale blue stripes in the figure.

While this is possible for $2 f_{0}$ by choosing a relatively low thickness resonator, for what concerns $3 f_{0}$, there is a TE mode which is independent of $2 d$ and exactly at $3 f_{0}$. This implies that the second harmonic will be more critical and that it will be impossible to handle it by resorting only to the resonator shape factor.

Figure 2 shows a practical CDRA setup. The resonator, whose height is halved to $d$, is placed on a ground plane and slot-coupled to a feeding microstrip. The dimensions of the setup are given in Table 1, the substrate has $\varepsilon=10.2$, and 


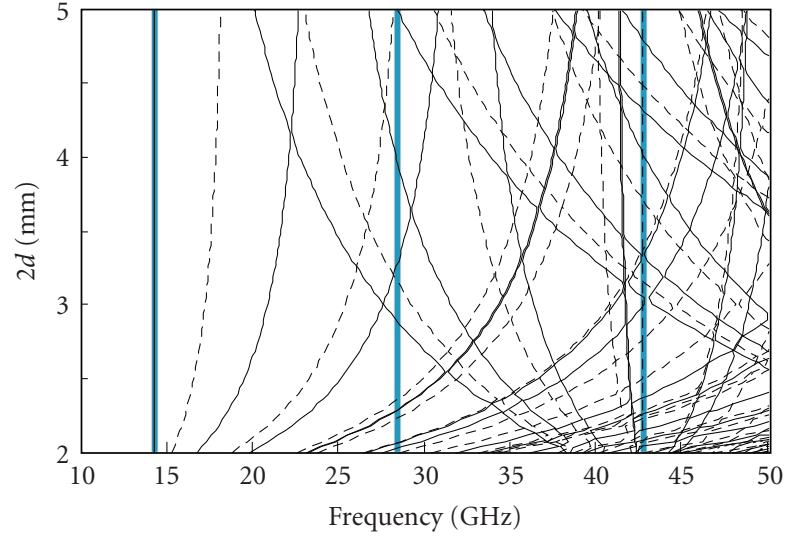

FIGURE 1: Curves of the resonant frequencies of the modes of the CDRA under analysis, as a function of resonator height having let radius to vary so that the first resonance remains fixed at $14.25 \mathrm{GHz}$. TE modes: solid lines; TM modes: dashed line.

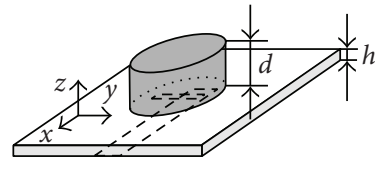

(a)

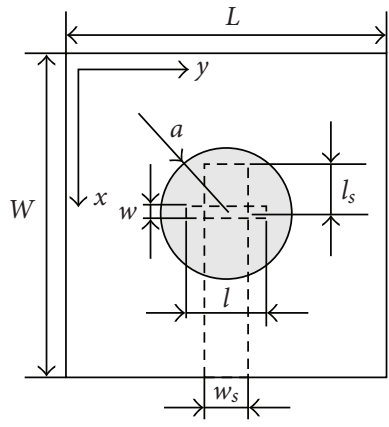

(b)
FIgure 2: Geometry of the CDRA under exam, top view (right) and $3 \mathrm{D}$ view (left).

TABLE 1: CDRA setup dimensions [millimeters].

\begin{tabular}{ccccccc}
\hline$W$ & $L$ & $t$ & $w_{s}$ & $l_{s}$ & $w$ & $l$ \\
\hline 16 & 16 & 0.635 & 4 & 2.88 & 0.7 & 4.7 \\
\hline
\end{tabular}

the whole set up has been analyzed with the finite elements method (FEM) in an enclosing box featuring perfectly matched layer (PML) radiation boundary conditions.

If a parametric analysis is performed over an appropriate range for $d$ and over the whole $f=10-45 \mathrm{GHz}$ frequency range, with the values of $a$ computed by (2), the return loss at the microstrip port can be represented as a surface graph function of $d$ and $f$. Figure 3 shows such a surface as a contour plot. Only the lines where $\left|S_{11}\right|$ is equal to -10 and $-20 \mathrm{~dB}$ are reported for the sake of clearness.

It is evident from Figure 3 how the fundamental mode is well matched for any $d$ value, even if the full wave analysis of the DRA over a finite ground leads to a slight dependence of the matching frequency with $d$ (i.e., the zone of matching is not a vertical line). Then there are zones, at higher frequencies, where the antenna is more or less matched, and these zones assume a form which is quite similar to the lines

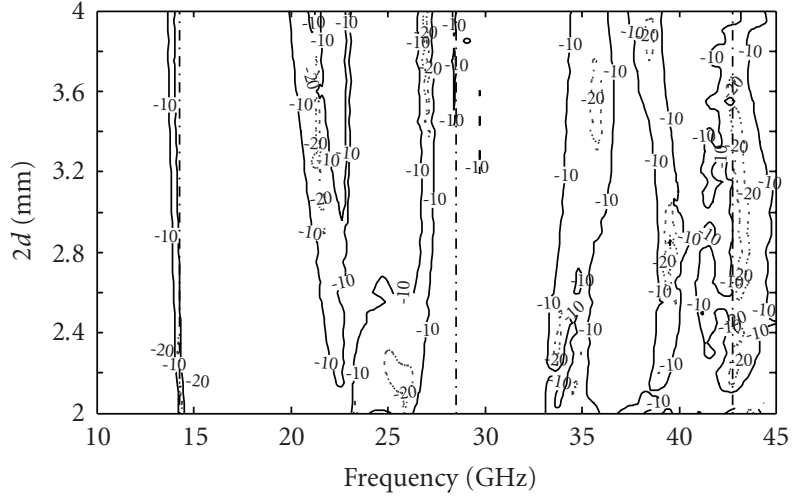

FIGURE 3: Contour plot of the magnitude of the $S_{11}$ parameter at the microstrip port of the antenna.

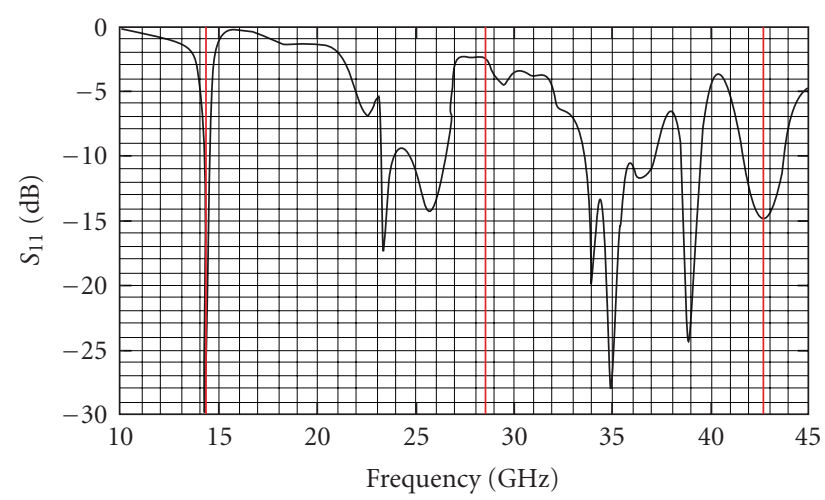

FIGURE 4: Return loss numerically computed for the CDRA setup of Figure 2. Good matching at the fundamental frequency is evident, while mismatch at first and second higher-order harmonics is insufficient.

in the dispersion graph in Figure 1. Lines are less numerous than in Figure 1 due to the fact that the coupling slot, due to its symmetries, does not excite all the possible modes and that matching has its own band, so neighboring lines easily merge in a single matched area. Around $2 f_{0}$ the reflection coefficient amplitude is high for low $d$ and gets lower as $d$ increases, confirming that, a low $d$ value is desirable. In the end, as expected, the reflection coefficient at $3 f_{0}$ is generally quite low. As a first conclusion a $d=1 \mathrm{~mm}$ will be the first choice, and, for this value of the resonator height, in Figure 4 the return loss as a function of the frequency is reported from $10 \mathrm{GHz}$ to $45 \mathrm{GHz}$, to show the antenna behavior at fundamental frequency and at first and second harmonics.

\section{Slot Harmonic Suppression}

Working only on the shape factor does not lead to a complete control over the reflection coefficient at higher harmonics. The $3 f_{0}$ one in particular being extremely critical as the previous section showed.

As an upgrade to the previously shown configuration a parasitic element has been inserted in the coupling slot, in a fashion similar to $[7,8]$. It is worth noticing how the "T" 


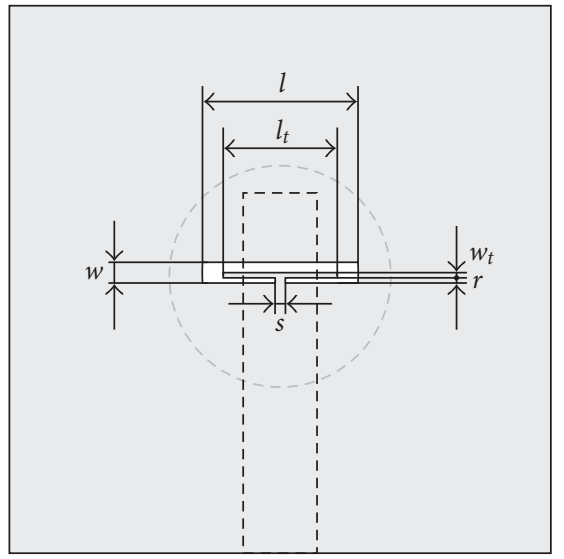

FIGURE 5: Geometry of the modified slot coupling. Inside the slot a parasitic reactive dipole provides higher harmonic rejection.

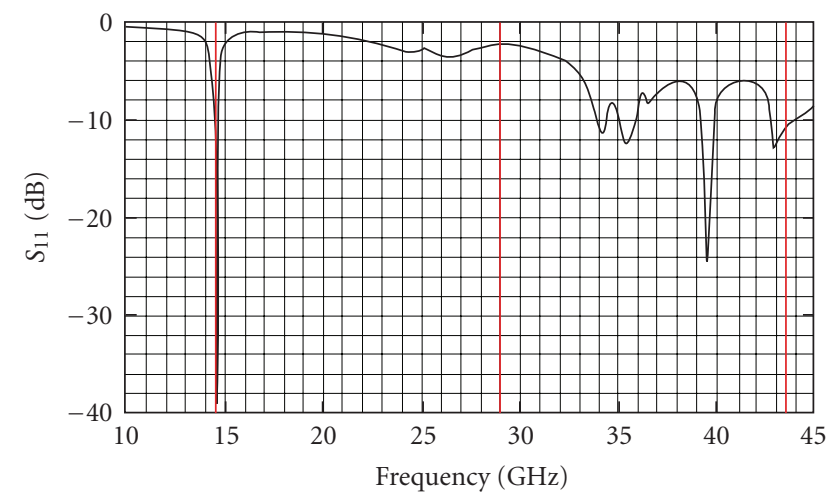

FIGURE 6: Return loss of the modified structure exhibiting the inslot parasitic element as sketched in Figure 5.

shape metalization inserted has to be designed differently since the slot itself is not resonant in our case. In Figure 5 the geometry of the proposed structure is reported. Dimensions are $w=0.6 \mathrm{~mm}, w_{t}=0.26 \mathrm{~mm}, l=6.3 \mathrm{~mm}, l_{t}=5.48 \mathrm{~mm}$, $r=0.34 \mathrm{~mm}$, and $s=1.1 \mathrm{~mm}$.

In Figure 6 the return loss as a function of the frequency is reported from $10 \mathrm{GHz}$ to $45 \mathrm{GHz}$, to show the antenna behavior at fundamental frequency and at first and second harmonics. For this structure the antenna input impedance is about $Z_{11}=45+j 5 \Omega$ at $f_{0}$ and $Z_{11}=85+j 28 \Omega$ at $2 f_{0}$.

A real full control of the second harmonic using the parasitic element is not possible, due to both the reduced size of the slot and a further phenomenon which will be described in detail in the following section. Hence, to further improve the antenna performances at the second harmonic, two parallel additional slots have been added to the previous configuration as shown in Figure 7, where $w=0.6 \mathrm{~mm}$, $w_{t}=0.26 \mathrm{~mm}, w_{a}=0.4 \mathrm{~mm}, l=6.3 \mathrm{~mm}, l_{t}=5.48 \mathrm{~mm}$, $l_{a}=2.0 \mathrm{~mm}, r=0.34 \mathrm{~mm}, d_{1}=2.8 \mathrm{~mm}, s=1.1 \mathrm{~mm}$, and $d_{2}=1.4 \mathrm{~mm}$.

In Figure 8 the return loss as a function of the frequency is reported from $10 \mathrm{GHz}$ to $45 \mathrm{GHz}$, to show that the antenna behavior at the second harmonics has been improved. In

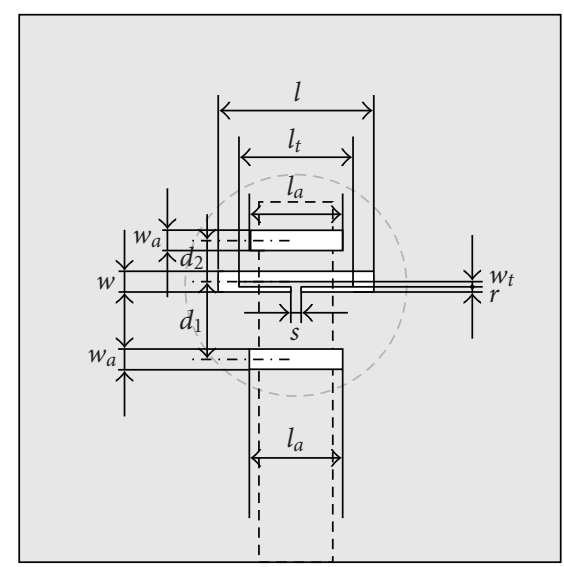

FIGURE 7: Geometry of the modified slot coupling with two additional parallel slots.

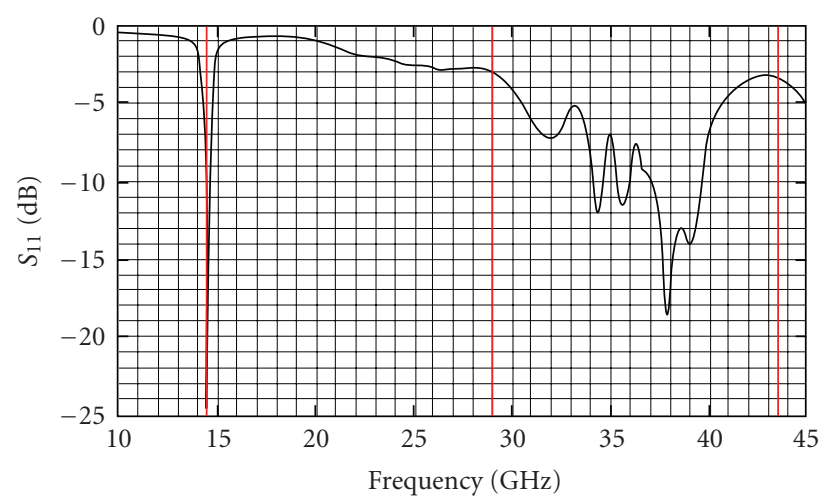

FIGURE 8: Return loss of the modified structure with two additional parallel slots as sketched in Figure 7.

particular for this modified structure the antenna input impedance is about $Z_{11}=50+j 5 \Omega$ at $f_{0}, Z_{11}=10+j 17 \Omega$ at $2 f_{0}$ and $Z_{11}=35+j 25 \Omega$ at $3 f_{0}$. The reason why the insertion of additional slots might help in rejecting higherorder harmonics lies in the fact that they perturb these more, due to their reduced wavelength. A deeper insight can be found in [12].

\section{Final Layout}

In this section a complete design ready to be integrated with a PA is presented. The design comprises not only the pad where the PA output is to be placed but also the line for the DC feed of the PA front-end. This latter has been designed so as to help in rejecting the second harmonic, while an additional stub shortening the third harmonic is also present. As shown in Figure 9, where the geometry of the final layout is reported, the parasitic element in the slot presented in the previous section has been retained, while the two additional slots have not, since their influence is relatively small and they can be replaced by the aforementioned stub. The geometric parameters defining the dimensions of the structure are as follows: $l=6.224 \mathrm{~mm}, w=611 \mu \mathrm{m}, l_{t}=5 \mathrm{~mm}, w_{t}=$ 


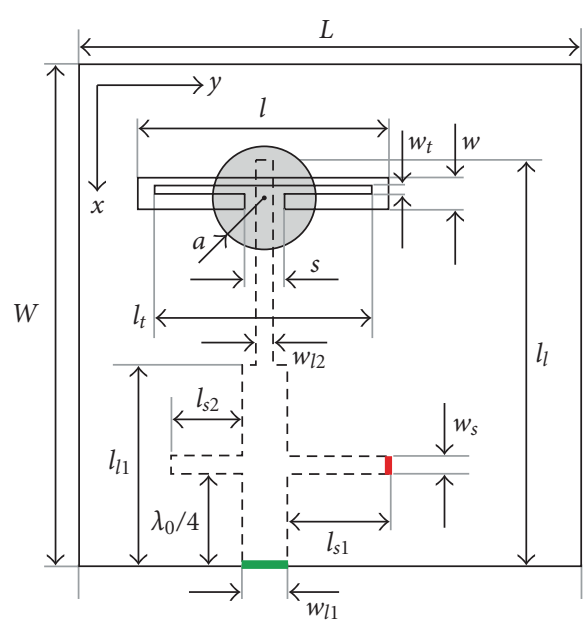

(a)

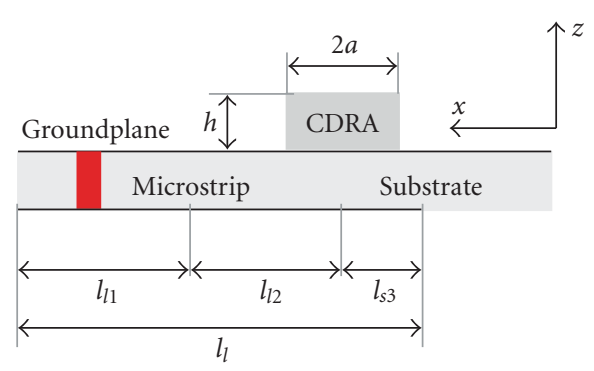

(b)

Figure 9: Geometry of the final layout: top view (a) and side view (b). The green rectangular area represents the DC feed, whereas the red rectangular pad represents the area where the PA output is to be soldered.

TABLE 2: Final layout input impedance.

\begin{tabular}{lcc}
\hline Frequency $[\mathrm{GHz}]$ & $Z_{11}[\Omega]$ & Requested $Z_{11}[\Omega]$ \\
\hline$f_{0}$ & $17.7+j 17.7$ & $15+j 18$ \\
$2 f_{0}$ & $21.5+j 94.71$ & $j 100$ \\
$3 f_{0}$ & $53.81+j 28.61$ & $j 17$ \\
\hline
\end{tabular}

$220 \mu \mathrm{m}, s=1.107 \mathrm{~mm}, a=1.39 \mathrm{~mm}, h=1.21 \mathrm{~mm}, l_{l 1}=$ $6.124 \mathrm{~mm}, l_{l 2}=8.876 \mathrm{~mm}, l_{s 1}=2.243 \mathrm{~mm}, l_{s 2}=1.754 \mathrm{~mm}$, $l_{s 3}=283 \mu \mathrm{m}, w_{l 1}=521 \mu \mathrm{m}, w_{l 2}=400 \mu \mathrm{m}, w_{s}=380 \mu \mathrm{m}$, and $\lambda_{0}=21.053 \mathrm{~mm}$ being $\lambda_{0}$ the wavelength along the microstrip at fundamental frequency $f_{0}$.

To show the active antenna electromagnetic performances in Figure 10 the input impedance is reported as a function of frequency, zooming around the fundamental frequency and the first two harmonics. Table 2 summarizes the input impedance simulation results at the above mentioned frequency together with the desired values as given by the PA designers [17].

In Figure 11 the electric field maps on the $y=0$ plane at fundamental frequency and at the first and second harmonics are represented. It is evident that the fundamental frequency radiates, while the second harmonic does not. This is due to the DC feed line for the power amplifier, which is loaded by the DC source which in turn is essentially a

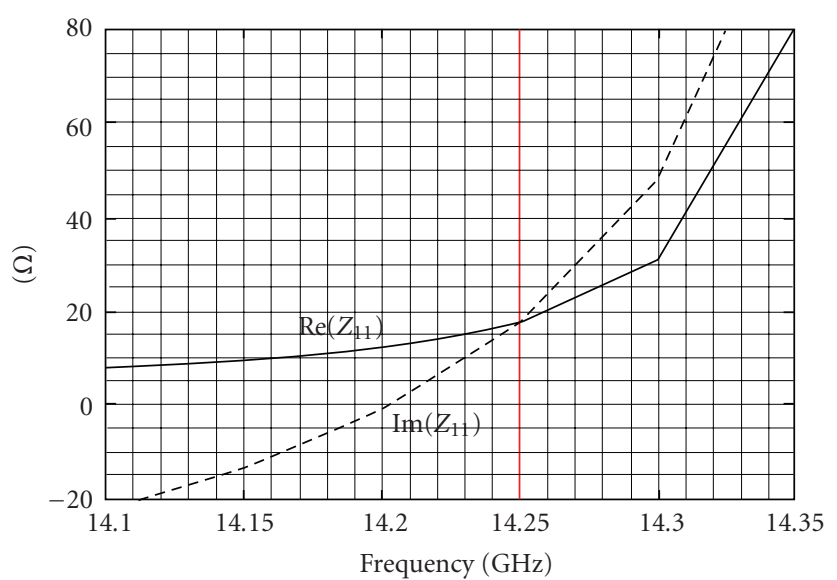

(a)

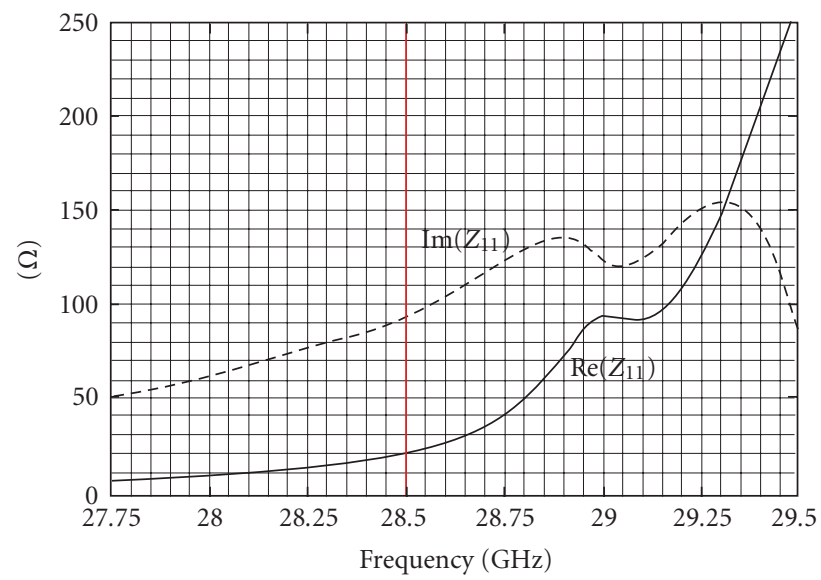

(b)

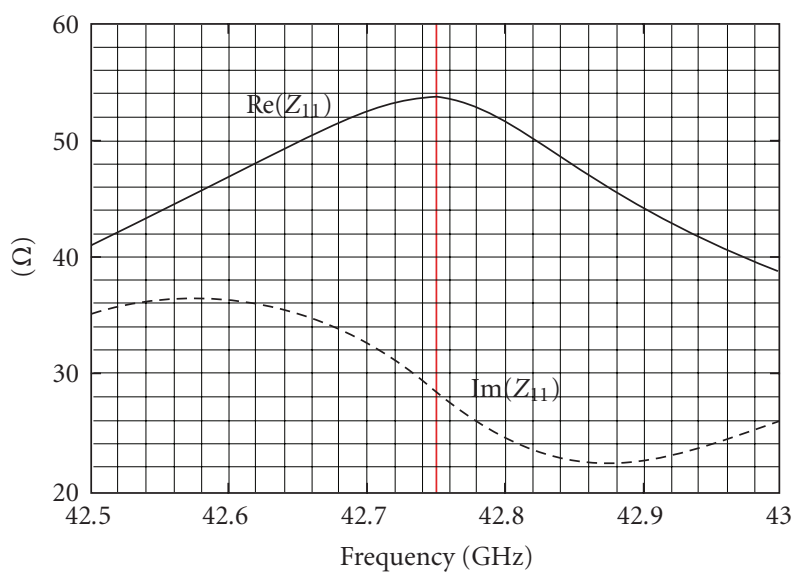

(c)

FIGURE 10: Final layout: input impedance at central frequency (a), at first harmonic (b), and at second harmonic (c).

short circuit at radio frequency. The feed is $\lambda_{0} / 4$ wavelength away from the PA output at $f_{0}$, hence the short circuit reverts to an open one and no energy is present on the stub at $f_{0}$ (see Figure 11(b)). On the other hand, at the first harmonic, $2 f_{0}$, the same stub is $\lambda / 2$ long and hence the PA output is almost shortened. This explains the strong standing wave 


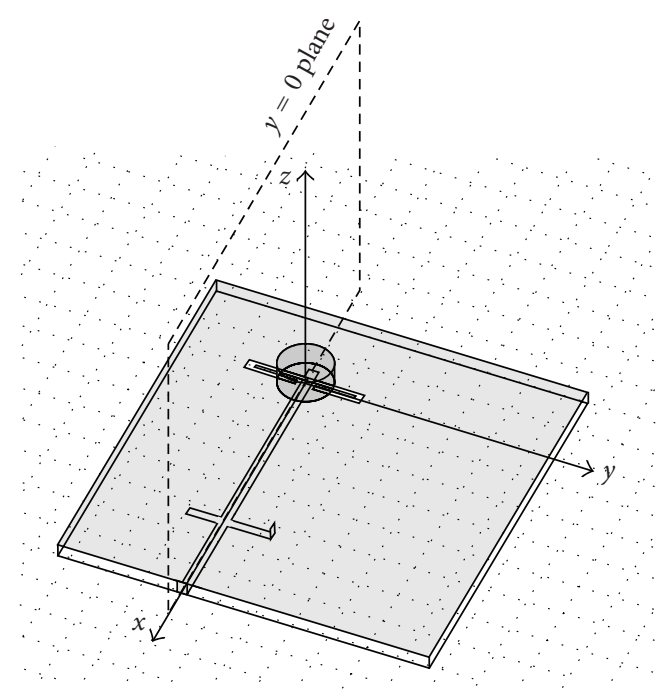

(a)

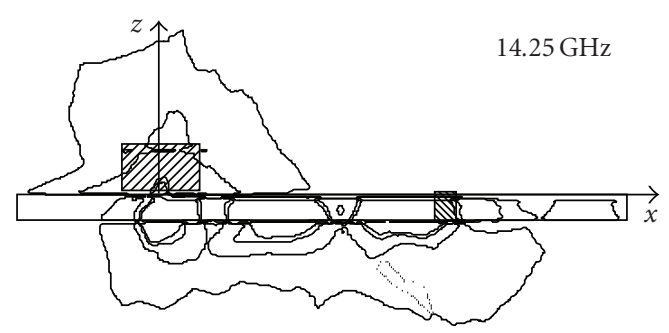

(b)

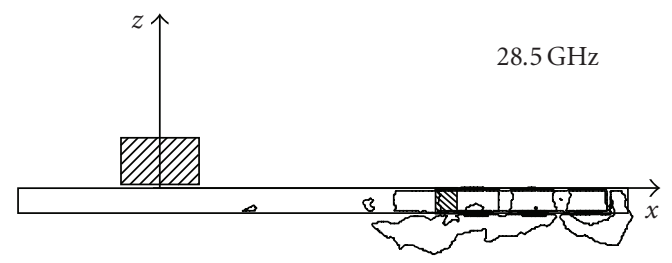

(c)

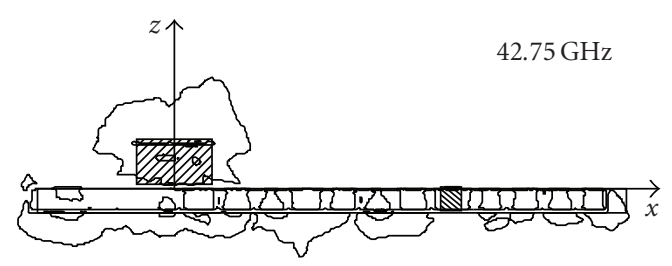

(d)

Figure 11: Final layout: (from top to bottom) observation plane geometry, electric field maps on $y=0$ plane at $14.25 \mathrm{GHZ}$, $28.5 \mathrm{GHz}$, and $42.75 \mathrm{GHz}$.

which can be seen in Figure 11(c). The issue still lies in the third harmonic because, even if the additional stub $l_{s 2}$ should shorten it, the slab is not thin with respect to $\lambda$ at $3 f_{0}$ hence surface waves can arise quite easily. Indeed the presence of a surface wave originating at the matching stub immediately below the coupling slot is very evident in
Figure 11(d). Radiation from both the CDRA and the openended feeding line stub accounts for the much higher real part of the third-order input impedance.

\section{Conclusions}

In this paper, a harmonic tuning technique for a dielectric resonator antenna working in $\mathrm{Ku}$ band at $14.25 \mathrm{GHz}$ has been described. As a first study a slot-coupled cylindrical DRA configuration has been considered, studying its harmonic response at a shape factor level, with particular attention to higher-order resonant modes, to devise a possible optimal shape factor.

As a second step of the study the basic resonator antenna layout has been modified by introducing a parasitic reactive element in the coupling slot to improve antenna performances at first and second harmonics. This configuration allows us to obtain a significant improvement of the antenna performances at first harmonic but a real full control of the second harmonic is not yet possible, both mainly due to the reduced size of the slot. Yet this issue is of minor importance due to the fact that the power generated by the PA at the second harmonic is a few percent of the total one.

At last, a complete design suitable for the integration with a PA has been presented, where the feeding line has been optimized as well to achieve full impedance matching at fundamental frequency and higher harmonics.

\section{Acknowledgments}

This work was partially supported by the Italian Ministry of Education, University and Research, under the PRIN research Project 005098437 "Active Integrated Antennas for High-Efficiency Mobile Terminals".

\section{References}

[1] K. Chang, R. A. York, P. S. Hall, and T. Itoh, "Active integrated antennas," IEEE Transactions on Microwave Theory and Techniques, vol. 50, no. 3, pp. 937-944, 2002.

[2] Y. Qian and T. Itoh, "Progress in active integrated antennas and their applications," IEEE Transactions on Microwave Theory and Techniques, vol. 46, no. 11, part 2, pp. 1891-1900, 1998.

[3] V. Radisic, Y. Qian, and T. Itoh, "Novel architectures for high-efficiency amplifiers for wireless applications," IEEE Transactions on Microwave Theory and Techniques, vol. 46, no. 11, part 2, pp. 1901-1909, 1998.

[4] S. Kwon, B. M. Lee, Y. J. Yoon, W. Y. Song, and J.-G. Yook, "A harmonic suppression antenna for an active integrated antenna," IEEE Microwave and Wireless Components Letters, vol. 13, no. 2, pp. 54-56, 2003.

[5] Y. J. Sung and Y.-S. Kim, "An improved design of microstrip patch antennas using photonic bandgap structure," IEEE Transactions on Antennas and Propagation, vol. 53, no. 5, pp. 1799-1804, 2005.

[6] M. J. Cryan, G. R. Buesnel, and P. S. Hall, "Analysis and control of harmonic radiation from active integrated oscillator antennas," IEEE Transactions on Microwave Theory and Techniques, vol. 50, no. 11, pp. 2639-2646, 2002. 
[7] H. Kim, K. S. Hwang, K. Chang, and Y. J. Yoon, "Novel slot antennas for harmonic suppression," IEEE Microwave and Wireless Components Letters, vol. 14, no. 6, pp. 286-288, 2004.

[8] H. Kim and Y. J. Yoon, "Microstrip-fed slot antennas with suppressed harmonics," IEEE Transactions on Antennas and Propagation, vol. 53, no. 9, pp. 2809-2817, 2005.

[9] A. Petosa, A. Ittipiboon, Y. M. M. Antar, D. Roscoe, and M. Cuhaci, "Recent advances in dielectric-resonator antenna technology," IEEE Antennas and Propagation Magazine, vol. 40, no. 3, pp. 35-47, 1998.

[10] R. K. Mongia and A. Ittipiboon, "Theoretical and experimental investigations on rectangular dielectric resonator antennas," IEEE Transactions on Antennas and Propagation, vol. 45, no. 9, pp. 1348-1356, 1997.

[11] A. A. Kishk, "Dielectric resonator antenna elements for array applications," in Proceedings of IEEE International Symposium on Phased Array Systems and Technology (PAST '03), pp. 300305, Boston, Mass, USA, October 2003.

[12] A. Guraliuc, G. Manara, P. Nepa, G. Pelosi, and S. Selleri, "Harmonic tuning for Ku-band dielectric resonator antennas," IEEE Antennas and Wireless Propagation Letters, vol. 6, pp. 568-571, 2007.

[13] A. Guraliuc, G. Manara, P. Nepa, G. Pelosi, and S. Selleri, "Investigation on harmonic tuning for active $\mathrm{Ku}$-band rectangular dielectric resonator antennas," International Journal of Antennas and Propagation, vol. 2008, Article ID 437538, 6 pages, 2008.

[14] L. Lucci, G. Manara, P. Nepa, G. Pelosi, L. Rossi, and S. Selleri, "Harmonic control in cylindrical DRA for active antennas," in Proceedings of IEEE Antennas and Propagation Society International Symposium (APS '07), pp. 3372-3375, Honolulu, Hawaii, USA, June 2007.

[15] A. R. Guraliuc, L. Lucci, G. Manara, P. Nepa, G. Pelosi, and S. Selleri, "Investigation on mutual coupling level in DRA sub-arrays for Ku-band active integrated antennas," in Proceedings of IEEE Antennas and Propagation Society International Symposium (AP-S '08), pp. 1-4, San Diego, Calif, USA, July 2008.

[16] S. A. Long, M. W. McAllister, and L. C. Shen, "The resonant cylindrical dielectric cavity antenna," IEEE Transactions on Antennas and Propagation, vol. 31, no. 3, pp. 406-412, 1983.

[17] P. Colantonio, F. Giannini, R. Giofré, et al., "Active integrated antennas for efficient mobile terminals," in Proceedings of the 11th International Symposium on Microwave and Optical Technology (ISMOT '07), pp. 625-628, Rome, Italy, December 2007. 

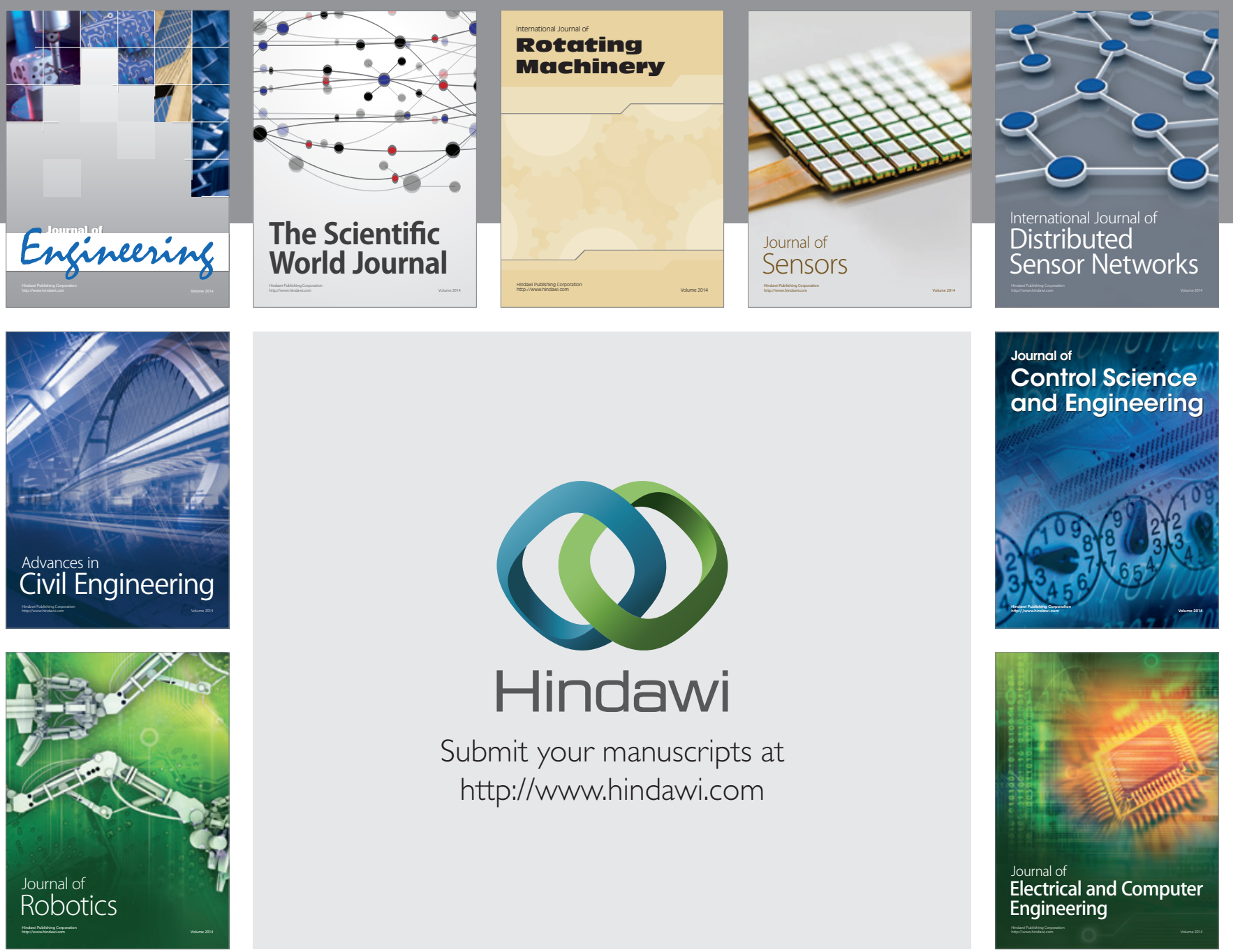

Submit your manuscripts at

http://www.hindawi.com
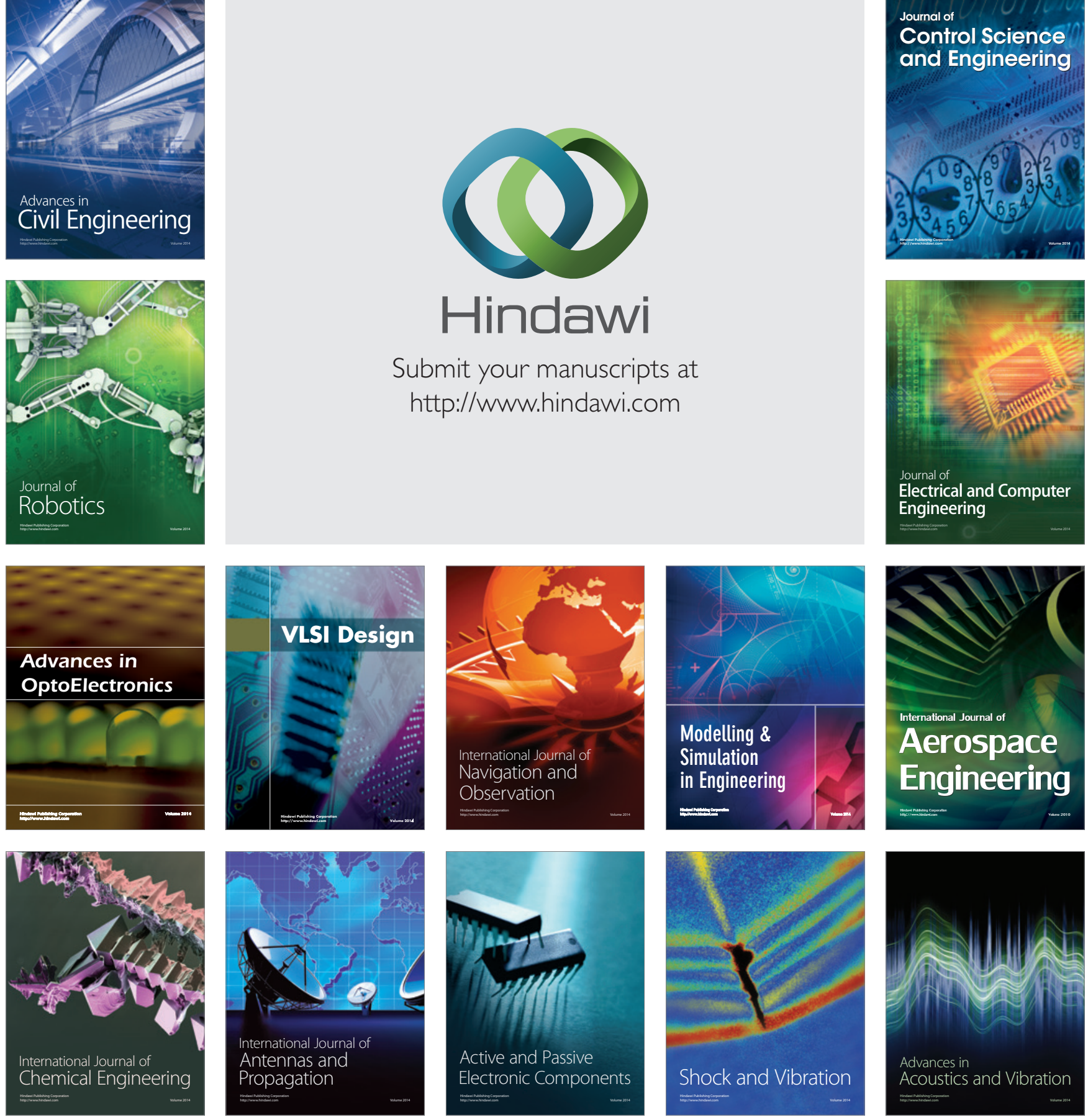